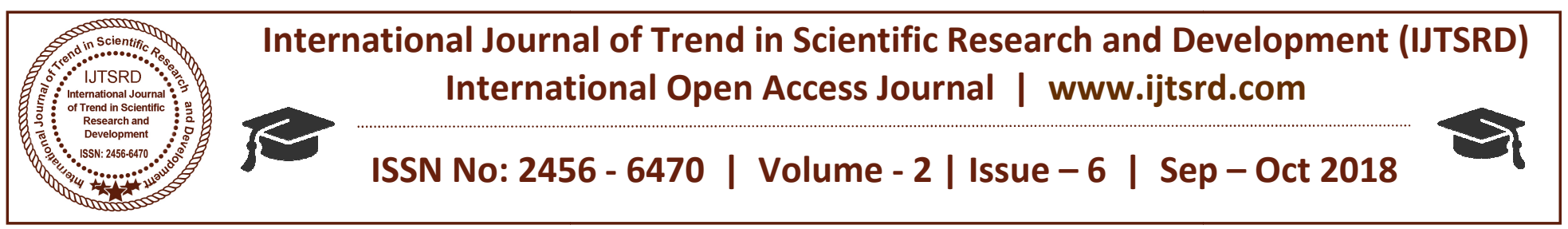

\title{
A Comparison Study Between Two Hydrogen Sensors
}

\author{
Dr. Albashir Zomrawi \\ Assistant Professor, College of Engineering, Karary University, Khartoum, Sudan
}

\begin{abstract}
In this research work, a comparison study has been carried out between Palladium (Pd) coated PMMA (Poly Methyl Meth acryl ate) optical sensor and Palladium thin films sensor that have been prepared on glass substrates. PMMA were sputter coated with Palladium using a sputter coating facility offered by the He riot Watt University, during sputter coating the target (palladium) is showered with argon gas such that atoms from the target are ejected by the ionized gas and then deposited on the PMMA substrate. Palladium thin films have been prepared on glass substrates by vaporization deposition technique with annealing temperature is around $600^{\circ} \mathrm{C}$. Relative inertness of the sensors to relative humidity makes it specific to detect hydrogen at low concentration. Target environments for the proposed sensors are fuel cell cabinets, industrial process plants, submarines, nuclear fuel power generation and decommissioning plants.
\end{abstract}

Results showed that optical characteristics of the prepared sensors are highly sensitive, but their properties considerably vary when the measurements conducted in vacuum or in air. In the two sensors the response-recovery time of $\mathrm{Pd}$ materials to hydrogen gas characterized to be extremely short.

Keyword: Micro electro mechanical systems, Palladium, Poly Methyl Meth acryl ate (PMMA), Sensor, Spectrometer, Substrates, Thin-Film.

\section{INTRODUCTION}

Hydrogen, occupies aboutmorethan90\%ofthe atmosphere "is a highly flammable gas and will burn at concentrations aslowas4\%inair ${ }^{[1]}$. It is the lightest of elements and the smallest molecule; it, therefore, has the greatest endency to leak. Thus, for a process ss safety application, a hydro gen leak can be more dangerous ${ }^{[8]}$.Detection of hydrogen gas has been extensively investigated with various materials 1-3used

as the sensing material. A large number of hydrogen sensors based on palladium and palladium alloyed with other metals, are reported in the literature4-14 but to date there is no single optical sensor that has all the desired characteristics (such as low hydrogen concentration $(<1 \%)$ detection in the presence of humidity with a response time of a few seconds). Palladium has been the focus of most research groups 13,15 because hydrogen is not only highly soluble in palladium but palladium also shows a high selectivity to hydrogen. In the presence of hydrogen, adsorption occurs on the surface 16, 17 of the palladium and hydrogen dissociates into atoms. The hydrogen atoms penetrate the subsurface layers of the palladium metal and palladium hydride is formed 11,18 . The adsorption of hydrogen in the palladium lattice leads to the expansion of the lattice but is dependent on the concentration of hydrogen and the working temperature [3]. Hydrogen sensors exploiting the Palladium/Palladium Hydride reaction are affected by humidity, whether 6 based on optical or electronic/catalytic detection, which poses difficulties for operation in field or real life conditions. Gases being monitored require pre-conditioning such as drying, to facilitate sampling at low humidity, and the sensor coating requires heating to maintain it at a constant temperature ${ }^{[4]}$.

\section{MECHANISMS FOR HYDROGEN SENSING}

Hydrogen sensor technology needs to satisfy three basic requirements. These requirements are; sensitivity, selectivity, and specificity ${ }^{[7]}$.

Different approaches used to sense and detect hydro gen. Numbers of which used in industry such as gas chromatography (GC), mass spectrometry (MS), catalytic bead (CB), and thermal conductivity.

Semiconducting metal oxide and CB sensors are popular solid-state technologies, these, sensors 
employ heated catalysts to sense hydro gen. They require e heating to about $300^{\circ} \mathrm{C}$ to enable surface actions that promote hydro gen sensing. Electrochemical sensors are based on known electrolytic reactions of hydro gen. Sensors based on catalytic combustion are generally non specific, electrochemical hydro gen sensors with liquid or solid type electrolytes having leakage issues. The hydro gen sensors based on thermal conductivity, CB, metal oxide, and electrochemical technologies require the presence of oxygen for sensor operation. Oxygen plays a crucial role in promoting the grain boundary formation in metal oxide sensor sand electron transfer reactions in electrochemical sensors.

The most promising solid-state technology is based on a hydrogen-specific material, palladium, which does not require oxygen for operation. Palladiumbased sensors are gaining wide popularity in the industry due to their reliability and high specificity to hydrogen.

\section{TRADITIONAL APPROACHES HYDROGEN SENSING}

TO

Number of traditional approaches can be used to detect Hydrogen, these may include:

a. Thermal Conductivity (TC): is the most widely applied measuring principle for the determination of hydrogen. The measuring principle is based on the differences in thermal conductivity of the gases to be measured. A Thermal Conductivity Detector (TCD) measures the concentration of a gas in a binary gas mixture by measuring the thermal conductivity of the sample gas and comparing it to the thermal conductivity of a selected reference gas ${ }^{[7]}$.

b. Gas Chromatography (GC): is also another widely applied measuring principle for hydrogen detection. The disadvantages of $\mathrm{G}$ Care long response times (minutes) due to the chromatography, time-intensive sample preparation, consumable (carrier and calibration gases), and lab or-intensive handling procedures. An advantage, however, is the ability to measure other gases such as nitrogen, oxygen, and carbon dioxide in the presence of hydrogen. But, this adds time to the total analysis ${ }^{[7]}$.

\section{SOLID-STATE APPROACHES TO HYDRO GEN SENSING}

A wide variety of solid-state sensors based on hydrogen-specific palladium, Metal Oxide
Semiconductor (MOS), CB, electrochemical, and Surface Acoustic Wave (SAW) technology are used in the industry for several years. Micro electro mechanical systems (MEMS) and nanotechnologybased devices for the measurement of hydro gen are there cent developments. These developments are mainly driven by the demands of the fuel cell industry. Solid-state approaches are gaining rapid popularity with in the industry due to their low cost, low maintenance, replacements, and flexibility of multiple installations with minimallabor.

'Hydrogen-Specific Palladium-Based Sensors are of three major classes of palladium-based hydrogen sensors' [3]. The most popular class of palladiumbased sensors is based on palladium resistors. A thin film of palladium deposited between two metal contacts shows a change in conductivity on exposure to hydrogen due to the phase transition in palladium. The palladium Field- Effect Transistors (FETs) or capacitors constitute is the second class, wherein the sensor architecture is in a transistor mode or capacitor configuration. The third class of palladium sensors includes optical sensors consisting of a layer of palladium coated on an optically active material that transforms the hydrogen concentration to an optical signal.

\section{TYPES OF PALLADIUM HYDROGEN SENSORS}

Several types of palladium-based hydrogen sensors have been reported in the literature. The most notable ones are based on Pd thin-film resistors, FETs, Pd nano wires, $\mathrm{Pd}$ nano particle networks, $\mathrm{Pd}$ nano clusters, and Pd nano tubes. Palladium Field-Effect Sensors: "Hydrogen sensors based on the "field effect" of palladium have been investigated extensively in the literature' [9] 'The field effect results due to the rapid dissolution of hydrogen in the palladium surface arranged in a $\mathrm{Pd}-\mathrm{SiO} 2-\mathrm{Si}$ configuration. The sensor relies on an electric field resulting from the charge transfer between palladium and hydrogen on its surface. The FETs' [7] and 'metal-insulatorsemiconductor (MIS)' ${ }^{[10]}$ are the two major types of device structures that have been studied for palladiumbased hydrogen sensing. Palladium is catalytically active, permeable to hydrogen, and can be readily used in FET and MIS devices.

a. Palladium-Based Resistors: 'Thick- and thin-film palladium-based resistors have been reported for hydrogen sensing' ${ }^{[6]}$. The thick-film device uses printed palladium paste on a ceramic substrate in 
a four- resistor net work. Two opposed resistors are covered to isolate them from the ambient atmosphere. 'The exposure of the uncovered resistors to hydrogen results in a change in resistivity of the thick-film material and a shift in the balance point of the bridge, which can be scaled to the hydrogen concentration. The thinfilm device is equivalent in design to the thick film; here, much thinner films (typically vacuum deposited) are used as the resistors. Thin-film palladium detectors have been prepared by depositing palladium through electron beam evaporation' [11], 'RF magnetron sputtering' [5], 'micro con-tact printing' [12], and wet electrochemistry. Most palladium resistors have fouling issues on the palladium surface due to impurities and pollutants in, or reaction with the air. The fouling on the palladium surface can be reduced by the addition of a second metal (alloy) to palladium.

b. B. Palladium-Coated Fiber Optic Sensors: A fiber optic hydro gen sensor consist sofa palladium coat in gat the en do fan optical fiber that senses the presence of hydro gen in air. When the coating reacts with the hydro gen, its optical properties are changed. Light from a central electro-optic control unit is projected down the optical fiber where it is either reflected from the sensor coating back to central optical detector or is transmitted to another fiber leading to the central optical detector. A change in the reflectedortransmittedintensityindicatesthepresenc eofhydrogen. Thefiberopticdetectoroffersinherents afetyby removing all electrical power from the test sites and reduces signal-processing problems by minimizing electromagnetic in te reference. The fiber optic hydro gen sensor scan be fabricated using a palladium-coated single-mode tapered optical fiber ${ }^{[12]}$. The attenuation change of the fiber-mode when the device was exposed to hydro gen is used to detect and measure hydro gen concentration in gaseous atmospheres.

\section{EXPERIMENTAL SETUP}

The Palladium thin films sensor system consists basic ally of seven components including chamber, white photo diode, spectrometer, vacuum unit, gauge pressure, cylinder of hydro gen gas, and computer as shown in schematic ally in figure(1). Figure (2) illustrates the sensor setup ${ }^{[1]}$.

A sample of Palladium coated glass slide sensor prepared first to detect hydro gen, and tested by X-ray to cheek its crystallization. The coated glass substrate (Palladium thin films) placed inside the developed polymer square test chamber of $50 \mathrm{~mm}$ square base and of $75 \mathrm{~mm}$ height with the top removable cover. The effective volume of the chamber was 187500 $\mathrm{mm} 3$; it has an inlet to allow the test gas to flow in. White photo diode that represent the light source used in the sensor,

CCS Spectrometer used to measure transmitted light while vacuum unit used to evacuate the test chamber. The gauge pressure utilized to measure the current pressure of the chamber.

Cylinder of hydro gen gas of a known con cent ration assisted the flow through the test chamber during measurement. Recorded data can be processed by a $\mathrm{PC}$ as illustrated in the figure.

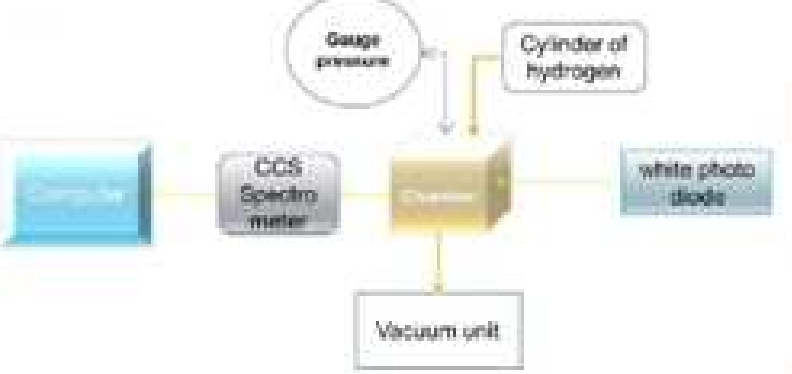

Fig.1: Palladium thin films sensor component

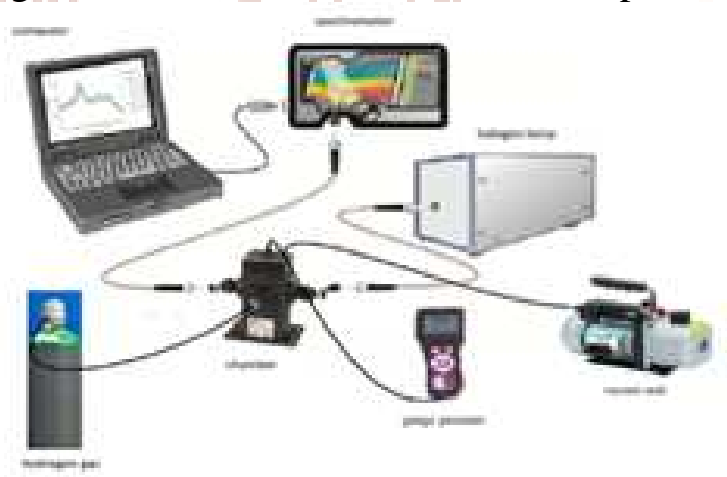

Fig.2: Palladium thin films sensor setup

On the other hand, Palladium coated (PMMA) optical sensor set up consisted of two gas cylinders [4]; a pure hydrogen cylinder and a carrier gas cylinder which contained either nitrogen or air. The coated PMMA substrate was placed inside a blacked out stainless steel chamber in which the gases were mixed. Transmission was measured using an Ocean Optics S2000 spectrometer and reflection was also measured using an Ocean Optics USB 2000 spectrometer. In all the experiments the light source used was a white tungsten halogen lamp. Figure (3) hereunder, shows a schematic diagram of PMMA optical sensor set up. 
The carrier gas was constantly cycled through the chamber and the system was allowed to equilibrate to this environment and the different concentrations of hydrogen gas were introduced in the system. The concentration of hydrogen and the level of relative humidity were monitored and controlled by using MKS 1179A mass flow controllers. After each different hydrogen concentration, the sensor was allowed to recover to initial condition in the carrier gas. Transmission and reflection spectra were recorded for each cycle of hydrogen and also a time acquisition spectrum was recorded to investigate the behavior of the sensor over time under the influence of varying hydrogen concentration and varying concentration of Relative Humidity.

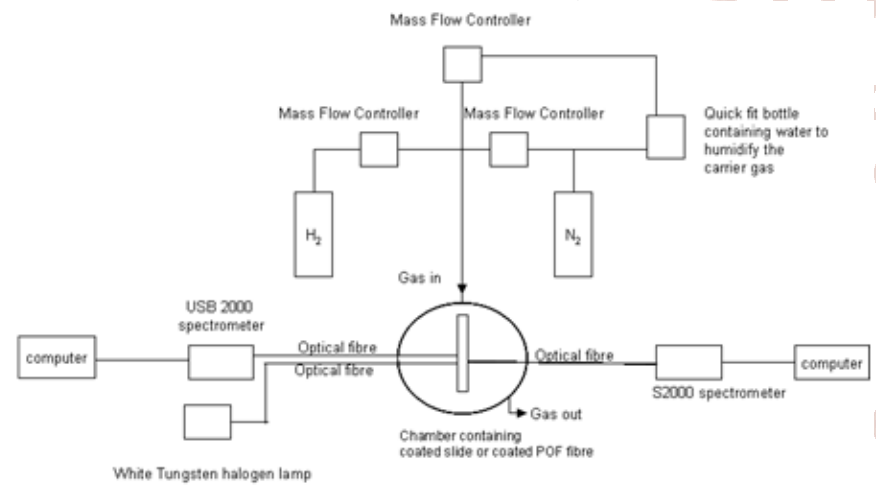

Fig.3: Experimental set up of Palladium coated PMMA optical sensor.

\section{MEASUREMENTS AND RESULTS}

In palladium thin film sensor, two testes were carried out to examine the sensors. In both tests, results were obtained through adopting the following steps:

a. Opening the test chamber to place the palladium thin film sensor on the sensor holder and close it.

b. The necessary light source was then directed by optical fiber and allowed to pass through the sample to the spectrometer.

c. The rotary pump then switched on to evacuate the test chamber to about -0.7 bars.

d. Next, the hydrogen gas of a known con cent rate ion allowed topass from the cylinder through the special inlet to the test chamber by opening the cylinder valve.

e. Test chamber pressure measured by observing the gauge pressure.

f. Spectrometer was then detected and analyzed transmitted signals and sent the data to the computer.

Numbers of measurements were carried out in different pressures. Six readings were observed for each sample test includingpressuresof-0.6,-0.5,-0.4,- 0.3,-0.2and-0.1bar. Frequency against Trans mitted light intensity graphs were produced for each reading. The six graphs were then combined to get her in one diagram with a back ground graph representing-0.7 pressure without Hydro gen.

Figure (4) and figure (5) below represent the resultant combinedgraphsPd1 and $\mathrm{Pd} 2$ for both sample tests.

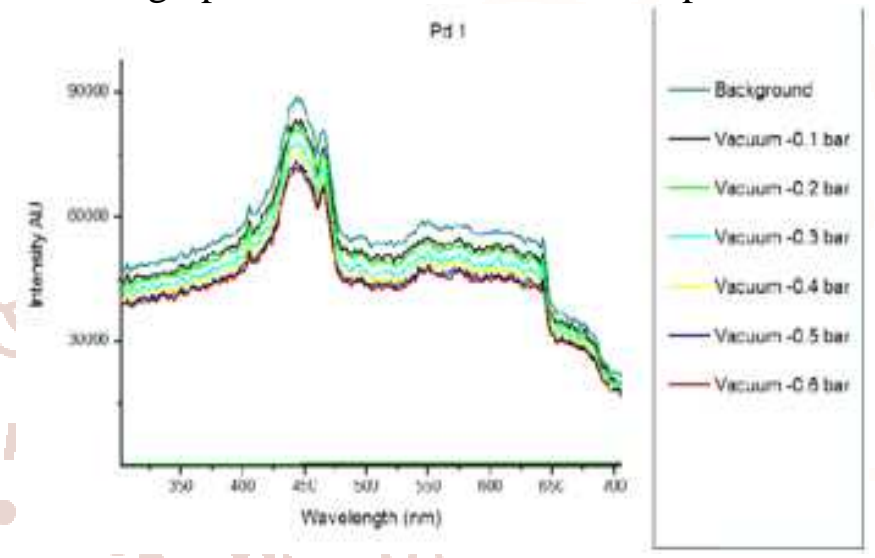

Fig.4: Combined graphs of the first sample.

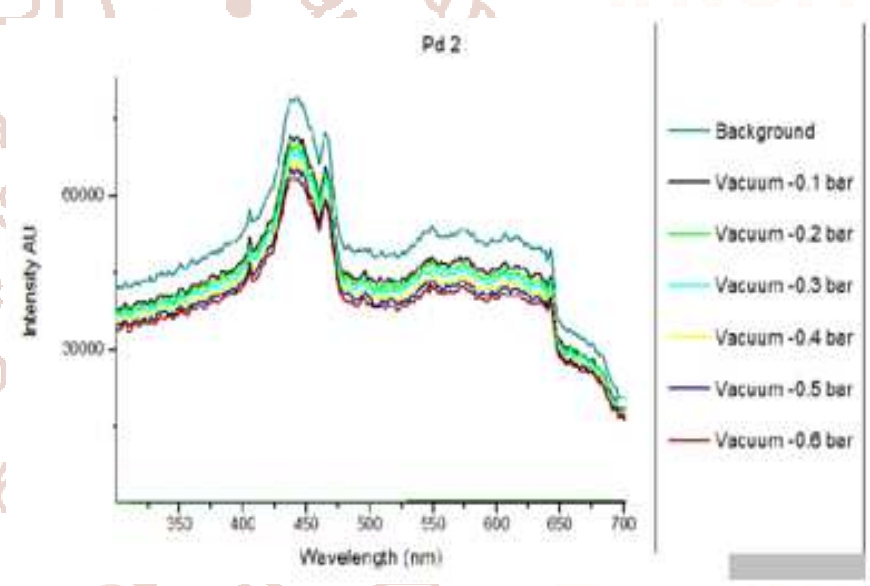

Fig.5: Combined graphs of the second sample.

From figures (4) and (5) obtain edbove it can be noted that by increase con cent ration of hydro gen gas in the test chamber, transmitted light is also increased that means the palladium thin film sample success fully detect hydro gen gas. Also, it can be noted that the suitable wavelength that can be used to detect hydro gen gas is a visible and in the regionof500$650 \mathrm{~nm}$.

For Palladium coated PMMA optical sensor a plot of transmitted spectral power against wavelength for varying concentration of relative humidity ranging from 0 to $100 \%$ plotted as shown in figure (6). The response of the sensor to relative Humidity was invariant as there was no change in transmitted spectral power for the different concentrations of Relative Humidity. 


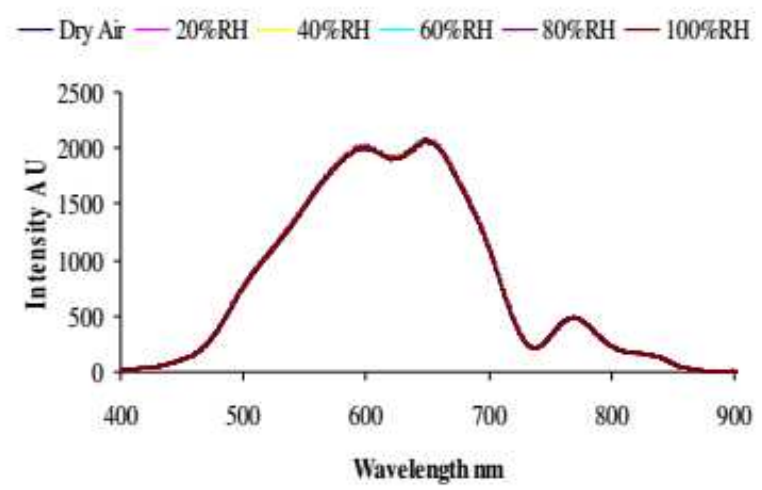

Fig. 6: The spectral power distribution at each Relative Humidity concentration.

Previous experiments showed that in the presence of relative humidity there is a $50 \%$ change in intensity of the transmission signal and it is difficult to distinguish between hydrogen detection and relative humidity. Results also showed that the proposed sensor operates unaffected by the relative humidity concentration. A possible explanation for the observed results was that the polymeric material used as the substrate, despite having hydrophilic carbonyl groups,

Figure (7) represents the transmitted and reflected spectral power distribution of a PMMA substrate sputter coated, and illuminated using a white tungsten halogen light source. The sensor was initially subjected to N2 to obtain a baseline, and then exposed to varying concentration of $\mathrm{H} 2$ while recovering in the carrier gas after each change in hydrogen concentration. The sensor had a low detection limit of $0.35 \% \mathrm{H} 2$. However the sensitivity limited by the mass flow controller and by the working temperature. The sensitivity of the sensor depended on the surface to volume ratio and it was observed that at $1 \% \mathrm{H} 2$ the sensor was at least twice as sensitive in reflection (526 AU/\%) as in transmission (219 AU/\%). Further experi ments shown that the sensor works faster in air than in nitrogen,
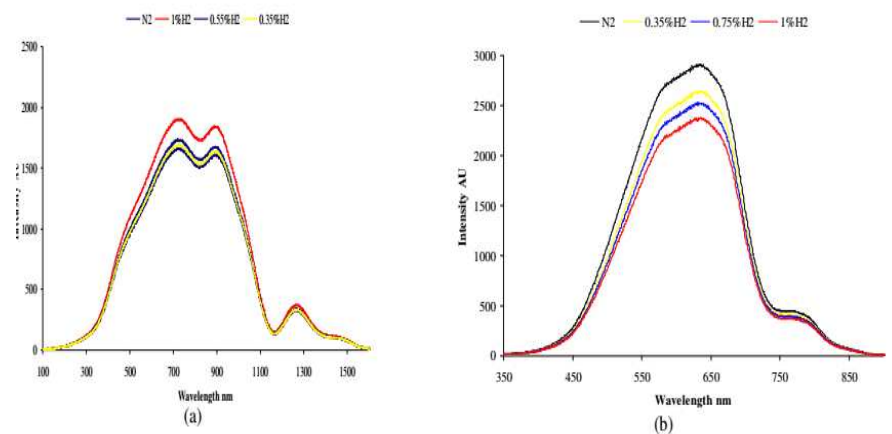

Fig.7: (a) transmitted spectral power distribution and

(b) reflected power distribution of a Palladium coated PMMA exposed to varying concentration of hydrogen.

\section{CONCLUSIONS}

This research work compares between Palladium (Pd) coated PMMA (Poly Methyl Meth acryl ate) optical sensor and Palladium thin films sensor that have been prepared on glass substrates. After analyzing results of each experiment carried out it was found to be that optical characteristics of the prepared sensors are highly sensitive, but their properties considerably vary when the measurements conducted in vacuum or in air. In the two sensors the response-recovery time of Pd materials to hydrogen gas characterized to be extremely short.

\section{REFERENCES}

1. Al basher Zomrawi, A. M. Awadelgied, S. F. Abdalah, K. Al Naimee, (2016), "Palladium Film Chemical Sensor", International Journal of Innovation in Science and Mathematics Volume 2, Issue 2, ISSN (Online): 2347-9051.

2. Cassidy, J., Pons, S. and Janata, J. (1986), "Hydrogen response of palladium coated suspended gate field effect transistor", Analytical Chemistry,58(8), 1757, 1986.

3. Lewis, F. A., "The Palladium Hydrogen System", Academic Press, London, 1967.

4. M.A.Nabeerasool1, P. J. Scully1, J. Vaughan1, R. Maier21ThePhoton Science Institute, the University of Manchester.

5. Oh, Y.-s., Hamagami, J. -i., Watanabe, Y., Takata, M. and Yanagida, H.(1993), "Palladium thin film hydrogen detector", Journal of the Ceramic Society of Japan, 101(1174), 618-620.

6. Pitts, J.R., Liu, P., Lee, S.-H. And Tracy, C.E.(2000), "Interfacial in stabilityin hydrogen sensors", Pro- ceedings of the DOE Hydrogen Program Review, Available at http://www1.eere. Energy. Gov/hydrogen and fuel cells/pd fs/28890g.

7. Ram B. Gupta (2009), "Hydrogen Fuel Production, Transport, and Storage". Edited by Taylor \& Francis Group, LLC.

8. Ram B. Gupta (2009), "Hydrogen Fuel Production, Transport, and Storage". Edited by Taylor \& Francis Group, LLC-2009.

9. Robins, I., Ross, J. F. and Shaw, J. E. A. (1986), "The logarithmic response of palladium-gate metal" insulator-silicon field-effect transistors to hydrogen, Journal of Applied Physics, 60(2), 843. 
10. Salomon's son, A., Eriksson, M. and Dan net UN, H. (2005), "Hydrogen interaction with platinum and palladium metal-insulator-semiconductor devices", Journal of Applied Physics, 98, 014505.

11. Thomas, R. C. and Hughes, R. C. (1997), "Sensors for Detecting Molecular Hydrogen Based on Pd Metal Alloys", Journal of Electrochemical Society, 144(9), 3245.
12. Villatoro, J., D' iez, A., Cruz, J. L. and Andre's, M.V., (2001), "Highly sensitive optical hydrogen sensor using circular Pd-coated single mode tapered fiber, Electron", 37, 1011.

13. Wolfe, D. B., Love, J. C., Paul, K.E., Chabinyc, M. L. And Whit esides, G. M., (2002), "Fabrication of palladium- based microelectronic devices by micro contact printing", Applied Physics Letters, 80, 2222.

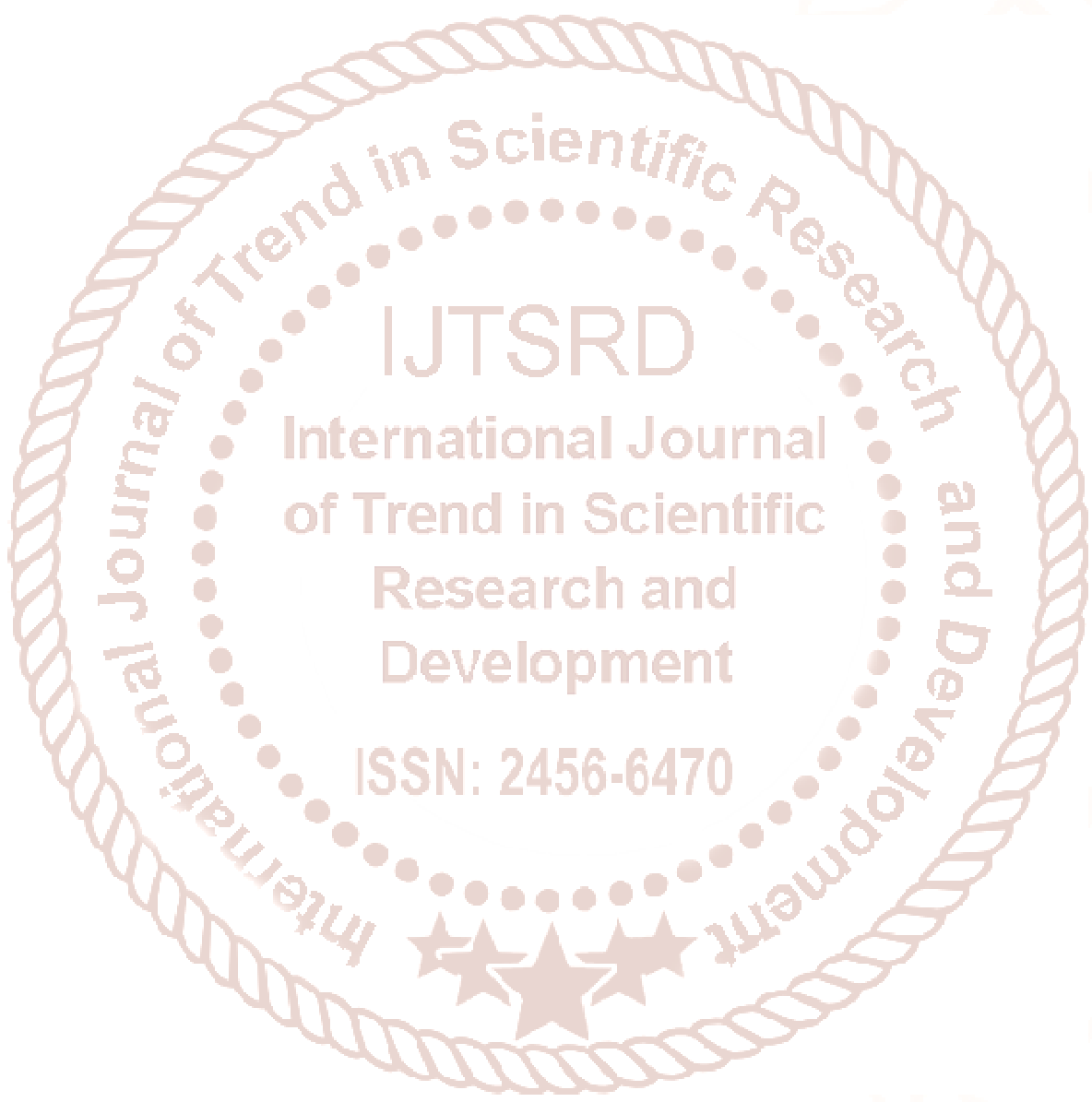

